УДК 628.477 .6

\title{
УТИЛИЗАЦИЯ ОТРАБОТАННОЙ ГЕРМЕТИЗИРУЮЩЕЙ ЖИДКОСТИ В СОСТАВЕ АСФАЛЬТОБЕТОННЫХ ПОКРЫТИЙ
}

\author{
Дюрягина Антонина Николаевна ${ }^{1}$, \\ adyuryagina@inbox.ru
}

\section{Бызова Юлия Сергеевна ${ }^{1}$, yuliyabyzovva@gmail.com}

\section{Островной Кирилл Александрович1, kostrovnoy@mail.ru}

\author{
Тюканько Виталий Юрьевич2, \\ vetal3333@mail.ru \\ 1 НАО Северо-Казахстанский университет им. М. Козыбаева, \\ Казахстан, 150000, г. Петропавловск, ул. Пушкина, 86. \\ 2 TOO «AVAGRO», \\ Республика Казахстан, 150000, г. Петропавловск, ул. Новая, 122.
}

\begin{abstract}
Актуальность настоящего исследования продиктована необходимостью решений в области вторичной переработки различных промышленных отходов с целью их использования в составе нового продукта. Объект исследования - отработанный герметик АГ-4И, который применяется большими объемами на объектах энергетики, нефттегазодобывающих предприятиях, машиностроительных заводах, городских коммунальных хозяйствах и других отраслях промышленности в системах теплофикации. Вторичная переработка экономит природные ресурсы, сокращает количество твердых отходов, уменьшает загрязнение воздуха и воды и вредные выбросы в атмосфреру. Дорожно-строительная отрасль может использовать переработанные материалы в составе асфральтобетонных покрытий. Модифицирование асфальтобетонных смесей отходами производства выступает в качестве приоритетного направления с целью сокращения огромного объема отходов, образующихся из различных источников.

Целью настоящей работы явилось исследование возможности утилизации отработанной герметизирующей жидкости АГ-4И в составе асфральтобетонных композиций с целью модифицирования.

Методы: реологические методы, структурно-механические методы оценки качества пленок и покрытий.

Результаты. Были определены физико-химические и реологические характеристики отработанной герметизирующей жидкости в составе битумных композиций. В результате проведенных исследований выявлено, что консервация исследуемой герметизирующей жидкости в предпочтительном твердом виде является невозможной, m. к. попьтки полимеризации полиизобутилена в составе отхода герметизирующей жидкости не привели к изменениям состава. Определено, что введение исследуемой герметизирующей жидкости в состав асфальтобетонных композиций позволяет использовать ее в качестве стабилизирующей и гидрофобизирующей добавки. Как следствие, улучшаются показатели адгезии к минеральным наполнителям и прочность покрытий.
\end{abstract}

\section{Ключевые слова:}

Утилизация отходов, герметизирующая жидкость, вторичное использование, асфральтобетонная смесь, битум.

\section{Введение}

Современные тенденции в области экологических проблем загрязнения окружающей среды диктуют острую необходимость приоритетных решений вторичной переработки различных промышленных отходов с целью их повторного использования [1-3]. Крупнотоннажные промышленные отходы, такие как отходы горнодобывающей, нефтяной и газовой промышленности нашли широкое применение в различных отраслях инфраструктуры $[4,5]$. Но, несмотря на это, большинство промышленных отходов традиционно утилизируются. Подход к утилизации предполагает уничтожение полезного продукта, при котором происходят невосполнимые потери ранее извлеченного минерального сырья, а процессы экологически безопасной нейтрализации отходов часто являются незавершенными. Данный подход к решению уничтожения отработанных отходов не является правильным, однако в значительной части случаев это объясняется дефицитом окупаемых технологий с завершенным циклом трансформации токсичных для окружающей среды соединений в технологически полезный продукт [6]. Главнейшим направлением сбережения ресурсов является применение вторичных материальных ресурсов, полученных из смешанных и оборотных отходов [7].

Анализ результатов научно-исследовательских работ $[8,9]$ доказывает, что различные виды отходов производства и потребления могут быть применены в качестве вторичного сырья с целью получения товаров технологического назначения и потребления. Возможность и целесообразность использования отходов доказана практикой работы предприятий различных отраслей промышленности [10]. Особенно востребованы они в производстве строительных и дорожно-строительных материалов. Большое количество экспериментальных исследований и разработок посвящено применению различных отходов в каче- 
стве модификаторов в составе асфальтобетонных композиций [11]. Одним из перспективных путей решения проблемы модифицирования асфальтобетонов является частичная замена дорогостоящих добавок более дешевыми отходами. Доказано, что производство модифицированных асфальтобетонных смесей с использованием вторичного сырья служит средством ограничения огромного объема отходов, образующихся из различных источников. Это также снижает потребление естественно добываемых материалов, следовательно, сводит к минимуму воздействие дорожной промышленности на окружающую среду [12-14].

Основными составляющими асфальтобетонных смесей являются битумы [13, 15-17], имеющие низкую стоимость и высокий спрос при производстве асфальтобетонных материалов. Основой для применения битумов при производстве асфальтобетонных материалов является способность формировать относительно прочные, гидрофобные (водоотталкивающие) пленки на поверхности бетонов, природных твердофазных наполнителей (щебень, песок) [18, 19]. По химическому составу битумы представляют собой сложную смесь углеводородов и гетероатомных соединений с молекулярной массой от 300 до 200010000. По растворимости (совместимости) отдельных составляющих битумов в органических растворителях в их составе выделяют следующие компоненты: масла (углеводороды), смолы, асфальтены [20, 21].

Неудовлетворительное качество асфальтобетонных битумных покрытий можно соотнести с нестабильностью и тенденцией к преобразованиям трехмерных битумных структур в результате влияния факторов окружающей среды [22-24]. Оптимизация количественных соотношений компонентов в битумном вяжущем путем введения в состав добавок различной природы является условием для образования устойчивых пространственных структур. В результате наблюдается стабилизация физико-химических, структурных, механических свойств в объемной фазе и на поверхности [25]. Значительный интерес с позиций модифицирования представляют синтетические битумы, что продиктовано присутствием в их составе соединений различной природы, способных хорошо физически и химически совмещаться с разнообразными полимерами, эластомерами, минеральными наполнителями, пластификаторами.

В настоящее время широко применяются полимербитумные, битум-каучуковые, битум-смоляные и другие композиции различного состава и назначения. Для улучшения реологических (структурных, жидкотекучих) характеристик битумов используют различные пластификаторы (церезин, петролатум, высоковязкие масла и некоторые сложные эфиры). Модификация битума такими полимерными добавками, как термоэластопласты, латекс, терполимеры, способствует улучшению свойств битума и повышению долговечности асфальтобетона. Данные добавки увеличивают когезионную прочность и термостойкость битума, а также придают эластичность, улучшают его поведение при низких температурах. Основной причиной, препятствующей широкому применению по- лимерных модификаторов, является их высокая стоимость. Кроме того, эти добавки должны отвечать двум требованиям. Во-первых, они должны быть совместимы с битумом, во-вторых - обеспечивать битуму требуемые улучшенные свойства [26, 27].

Основной целью данного исследования являлось установление возможности вторичного использования отработанной герметизирующей жидкости (ТУ 26-02-592-83 «Герметизирующие жидкости АГ-4И») в составе асфальтобетонных композиций. Данные герметизирующие жидкости в больших количествах используются для антикоррозионной защиты внутренней поверхности резервуаров на объектах энергетики, нефтегазодобывающих предприятиях, машиностроительных заводах, городских коммунальных хозяйствах и других отраслях промышленности в системах теплофикации. По истечении срока эксплуатации герметизирующая жидкость изымается, и, как следствие, возникает проблема ее утилизации.

\section{Основная часть}

На первом этапе представлялось целесообразным очистить отработанную герметизирующую жидкость от механических примесей. Удаление твердофазных частиц заключалось в предварительном подборе растворителя и растворении в нем отработанного герметика, центрифугировании и дальнейшей перегонке растворителя. Применение растворителя позволяет понизить вязкость отработанного герметика АГ-4И и увеличить скорость оседания частиц.

В качестве растворителей использовали традиционные ароматические углеводороды (ксилол), а также предельные и непредельные (сольвент, уайт-спирит) углеводороды, различающиеся по химическому строению, вязкости, температуре кипения, которые способны растворять и удерживать в объеме отдельные компоненты герметика, обеспечивать агрегативную устойчивость и требуемые реологические, адсорбционнокогезионные свойства в смесях (вязкость, растекаемость, способность удерживаться на поверхностях).

Одновременно, с учетом полимерного состава герметика, предусматривалось подобрать такой растворитель, который бы позволил целенаправленно регулировать прочность его структурной сетки, в том числе и за счет введения других веществ (поверхностно-активных, инициаторов полимеризации, сиккативов).

По результатам испытаний установлено, что ацетон, ксилол и бутилацетат не образуют с герметиком АГ-4И однородных смесей, расслаивание происходит уже на стадии приготовления смесей. С такими растворителями, как сольвент и уайт-спирит, герметик АГ-4И образует при любых объемных соотношениях идеально смешанные нерасслаиваемые в течение 6 суток системы. Аналогичную высокую растворимость герметика отмечали в технических растворителях - моторных топливах (бензины, дизельное топливо). С увеличением содержания герметика в этих растворителях условная вязкость смесей (по шариковому вискозиметру, $20{ }^{\circ} \mathrm{C}$ ) возрастает и при содержании АГ-4И свыше $15 \%$ стабилизируется на уровне 35-36 с. 
С увеличением концентрации герметика в сольвенте и уайт-спирите вплоть до 3,5-4,0 \% поверхностное натяжение $\left(\sigma_{\text {жг }}\right)$ смесей (по прибору Ребиндера) практически не изменяется. Дальнейшее увеличение содержаний АГ-4И сопровождалось структурообразованием полиизобутилена и, как следствие, увеличением натяжения пленок ( $\sigma_{\text {жг }}=54-60$ кДж/ $\left.\mathrm{m}^{2}\right)$. Формирование межмолекулярных сеток в объеме сопровождалось одновременно увеличением прочности пленок на разрыв - сил когезионного сцепления, со-

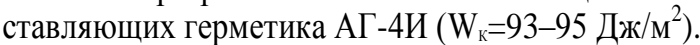

Таким образом, по результатам оценки физической совместимости герметика АГ-4И с растворителями, а также адгезионных и когезионных характеристик их пленок можно заключить, что для очистки отработанной герметизирующей жидкости предпочтительно использовать сольвент, уайт-спирит, бензины, дизельное топливо.

В исследованиях определяли физико-химические свойства отработанного герметика АГ-4И. В рамках лабораторных исследований установлено, что плотность образцов составила $880 \pm 5$ кг $/ \mathrm{M}^{3}$ (ГОСТ 18995.1-

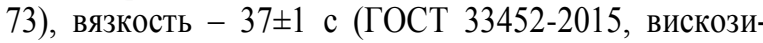
метр с падающим шариком, $20{ }^{\circ} \mathrm{C}$ ), температура вспышки - $155 \pm 3{ }^{\circ} \mathrm{C}$ (ГОСТ 4333-2014). По данным химического анализа в составе герметика не обнаружены механические примеси, кислоты, щелочи и вода. Основными составляющими герметика являются полиизобутилены, индустриальное масло, парафины. Парафины представлены смесью углеводородов нормального строения с 18-35 атомами углерода в молекуле с молекулярной массой 300-450. В естественных условиях $\left(18-25^{\circ} \mathrm{C}\right)$ из-за низкой летучести составляющих АГ-4И формирование пленок практически не наблюдается во времени (в течение 3-4 суток). Пленки герметика АГ-4И, полученные после его высокотемпературной обработки $\left(130-140{ }^{\circ} \mathrm{C}\right)$, отличаются от исходных незначительным снижением вязкости (32-34 с при $20{ }^{\circ} \mathrm{C}$ ), паропроницаемости (ГОСТ 32318-2012), температуры каплепадения (ГОСТ ISO

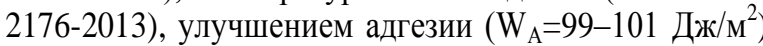
к стали (ГОСТ 15140-78). Последнее вызвано развитием процессов деполимеризации полиизобутилена при термической обработке герметика.

Введение в состав герметика традиционных инициаторов полимеризации (полиэтиленполиаминПЭПА, полиэтиленполиимин-ПЭПИ) в количествах 2-4 \% практически не оказало влияния на степень полимеризации полиизобутилена, как при $20{ }^{\circ} \mathrm{C}$, так и при повышенных температурах $\left(130-140{ }^{\circ} \mathrm{C}\right)$. Условная вязкость герметика в присутствии указанных инициаторов после предварительной термической обработки смесей возросла всего лишь на 2-3 с (при $20{ }^{\circ} \mathrm{C}$ ) и составила 39-41 с, а общая продолжительность сушки (формирование сухих пленок) практически не изменилась.

Были также предприняты попытки принудительного аминирования полиизобутилена в составе герметика АГ-4И аммиаком в присутствии кислотного и щелочного катализаторов при температурах 20, 50 и $100{ }^{\circ} \mathrm{C}$ в течение 1 ч. Однако данные физико- химического анализа показали, что материал остается практически неизменным как по реологическим свойствам (вязкость), так и по химическому составу.

Таким образом, консервация (захоронение) отработанного герметика АГ-4И не представляется возможной, так как попытки его предварительной полимеризации и термической обработки с целью перевода в твердофазную форму не дали положительных результатов. Последнее с учетом экологических нормативов на захоронение материалов потребует специальных герметичных коллекторов - хранилищ, что не совсем экономично и практично.

В этой связи предусматривалось оценить функциональную активность герметика АГ-4И в составах асфальтобетонных материалов по таким их эксплуатационным характеристикам, как влагопроницаемость и адгезия пленок, прочностные свойства покрытий и так далее.

Для проведения настоящих исследований был выбран вязкий нефтяной дорожный битум марки БНД 90/130, используемый для производства асфальтобетонных композиций. Были определены основные технические характеристики чистого битума и с добавками отработанного герметика АГ-4И, результаты представлены в табл. 1.

Таблица 1. Технические характеристики дорожного битума

Table 1. Technical characteristics of road bitumen

\begin{tabular}{|c|c|c|c|}
\hline \multirow[t]{2}{*}{$\begin{array}{l}\text { Наименование показателя } \\
\text { Name of parameter }\end{array}$} & \multicolumn{3}{|c|}{$\begin{array}{c}\text { Содержание отработанного герме- } \\
\text { тика АГ-4И (\% на массу битума) } \\
\text { Content of spent sealant AG-4I } \\
\text { (\% by weight of bitumen) }\end{array}$} \\
\hline & 0 & 2 & 5 \\
\hline $\begin{array}{l}\text { Глубина проникновения } \\
\text { иглы при } 25^{\circ} \mathrm{C} ; 0,1 \mathrm{mм} \\
\text { Needle penetration depth at } \\
25^{\circ} \mathrm{C} ; 0,1 \mathrm{~mm}\end{array}$ & 91 & 96 & 101 \\
\hline $\begin{array}{l}\text { Температура размягения } \\
\text { по кольцу и шару, }{ }^{\circ} \mathrm{C} \\
\text { Ring and ball softening } \\
\text { temperature, }{ }^{\circ} \mathrm{C}\end{array}$ & 75 & 62 & 60 \\
\hline $\begin{array}{l}\text { Растяжимость при } 25^{\circ} \mathrm{C} \\
\text { Extensibility at } 25^{\circ} \mathrm{C}\end{array}$ & 3,0 & 9,3 & 12,6 \\
\hline
\end{tabular}

Определено, что отработанный герметик хорошо растворим в битумном вяжущем (гомогенная смесь) в диапазоне 0-40\%. При модифицировании в концентрационном диапазоне свыше 10 \% битум приобретает свойства пластичности. Число пенетрации увеличилось практически вдвое. В дальнейших исследованиях ограничились модифицированием асфальтобетонных композиций отработанным герметиком до содержания последнего $5 \%$.

Оценены гидрофобные свойства модифицированных герметиком битумных составов путем измерения краевого угла смачивания (рисунок).

При низкой концентрации АГ-4И (до уровня $5 \%$ ) в битумных системах происходит ожидаемый пропорциональный прирост в краевых углах смачивания. Таким образом, ввод отработанного герметика улучшает водоотталкивающие характеристики битумов. 


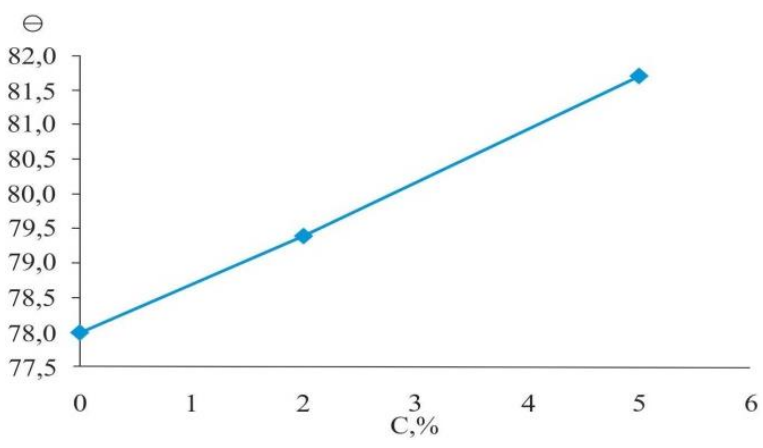

Рисунок.Зависимость краевого угла смачивания от концентрации герметика в битумных системах

Figure. Contact angle dependence on sealant concentration in bitumen systems

Дополнительно проведены испытания по определению качества сцепления битумного вяжущего в присутствии отработанного герметика с поверхностью щебня на основе кислых и основных горных пород (ГОСТ 12801-98). Для сравнительной оценки эффективности рекомендуемой адгезионной присадки (отход АГ-4И) испытания проводили в сопоставлении с показателями базового варианта, а именно без ис- пользования присадок и с применением традиционной адгезионной присадки, представляющей собой катионное поверхностно-активное вещество. Расходы адгезионных присадок варьировали в пределах от 0,2 до $2 \%$ (в пересчете на массу битума). Содержание традиционной присадки в битумной композиции задавали на постоянном уровне - 0,5 \%. Условия и показатели испытаний качества сцепления представлены в табл. 2.

При отсутствии добавок качество сцепления битума с гранитным щебнем неудовлетворительное и характеризуется двумя баллами. Хорошее качество (4 балла) сцепления битума на гранитном щебне достигнуто в присутствии рекомендуемой адгезионной присадки при ее расходе на уровне $1 \%$. Применение рекомендуемой адгезионной присадки, учитывая ее высокую гидрофобность, и одновременно достигнутое хорошее качество сцепления битумов с поверхностью щебня позволят уменьшить дополнительно водопроницаемость пленок битумного вяжущего. Таким образом, отработанная герметизирующая жидкость может быть рекомендована для использования в приготовлении асфальтобетонных смесей.

Таблица 2. Результаты определения сцепления вяжущего с поверхностью щчебня

Table 2. Results of evaluation of binder adhesion to the crushed stone surface

\begin{tabular}{|c|c|c|c|c|}
\hline \multirow{2}{*}{$\begin{array}{c}\text { Вариант } \\
\text { испытаний } \\
\text { Version of test }\end{array}$} & \multirow{2}{*}{$\begin{array}{c}\text { Наименование } \\
\text { адгезионной присадки } \\
\text { Adhesive additive } \\
\text { name }\end{array}$} & \multirow{2}{*}{$\begin{array}{c}\text { Количество } \\
\text { присадки, \% } \\
\text { Additive } \\
\text { quantity, \% }\end{array}$} & \multicolumn{2}{|c|}{$\begin{array}{c}\text { Качество сцепления на двух разновидностях щебня } \\
\text { Adhesion quality on two types of crushed stone }\end{array}$} \\
\hline & & & $\begin{array}{c}\text { Кислом/Acid } \\
\text { (гранитном/granite) }\end{array}$ & $\begin{array}{c}\text { Основном/Hydroxide } \\
\text { (доломитизированный/dolomite) }\end{array}$ \\
\hline \multirow[b]{2}{*}{ Базовый/Base } & $\begin{array}{c}\text { без присадки } \\
\text { without additives }\end{array}$ & 0 & $\begin{array}{c}\text { Плохое/Low } \\
\text { (два балла/two points) }\end{array}$ & $\begin{array}{c}\text { Хорошеe/Good } \\
\text { (четыре балла/four points) }\end{array}$ \\
\hline & $\begin{array}{c}\text { традиционная } \\
\text { присадка } \\
\text { traditional additive } \\
\end{array}$ & 0,5 & $\begin{array}{c}\text { Удовлетворительное } \\
\text { Satisfactory } \\
\text { (три балла/three points) }\end{array}$ & $\begin{array}{c}\text { Удовлетворительное Satisfactory } \\
\text { (три балла/three points) }\end{array}$ \\
\hline \multirow{4}{*}{$\begin{array}{l}\text { Рекомендуемый } \\
\text { Recommended }\end{array}$} & \multirow{4}{*}{$\begin{array}{c}\text { отработанная } \\
\text { герметизирующая } \\
\text { жидкость } \\
\text { waste sealing liquid }\end{array}$} & 0,2 & $\begin{array}{c}\text { Плохое/Low } \\
\text { (два балла/two points) }\end{array}$ & $\begin{array}{c}\text { Плохое/Low } \\
\text { (два балла/two points) }\end{array}$ \\
\hline & & 0,5 & $\begin{array}{c}\text { Удовлетворительное } \\
\text { Satisfactory } \\
\text { (три балла/three points) }\end{array}$ & $\begin{array}{c}\text { Удовлетворительное Satisfactory } \\
\text { (три балла/three points) }\end{array}$ \\
\hline & & 1,0 & $\begin{array}{c}\text { Хорошеe/Good } \\
\text { (четыре балла/four points) }\end{array}$ & $\begin{array}{c}\text { Хорошеe/Good } \\
\text { (четыре балла/four points) }\end{array}$ \\
\hline & & 2,0 & $\begin{array}{c}\text { Хорошеe/Good } \\
\text { (четыре балла/four points) }\end{array}$ & $\begin{array}{c}\text { Хорошеe/Good } \\
\text { (четыре балла/four points) }\end{array}$ \\
\hline
\end{tabular}

\section{Выводы}

1. Консервация исследуемой герметизирующей жидкости в предпочтительном твердом виде является невозможной, т. к. попытки полимеризации полиизобутилена в составе отхода герметизирующей жидкости не привели к изменениям состава.

2. Хорошая растворимость в битумсодержащих асфальтобетонных материалах отработанного герметика открывает возможности для применения

\section{СПИСОК ЛИТЕРАТУРЫ}

1. Сагдеева Г.С., Патракова Г.Р. Переработка отходов производства и потребления с использованием их ресурсного потенциала // Вестник Казанского технологического университета. 2014. - № 1. - C. 194-198.

2. Пинаев В.Е. Состояние и проблемы использования промышленных твердых отходов в России // Исследовано в России. 2004. - № 7. - C. 1397-1433. последнего в качестве водоотталкивающей и стабилизирующей добавки.

3. Доказано, что отработанная герметизирующая жидкость в качестве адгезионной присадки в асфальтобетонных смесях является эффективной в сравнении с показателями базового варианта с позиций улучшения качества сцепления битума с поверхностью минерального наполнителя.

3. Мазуркин П.М., Солдатова В.А. Современные проблемы совместной переработки твердых бытовых и промышленных отходов // Современные проблемы науки и образования. 2008. - № 6. - C. 59-68.

4. Косулина Т.П., Кононенко Е.А., Цокур О.С. Утилизация нефтяных шламов реагентным методом и использование продуктов утилизации в качестве вторичных материальных ресурсов // Альтернативная энергетика и экология. - 2012. № 2. - C. 187-192. 
5. Alkali-activated slag binders from rock-wool production wastes / V.T. Erofeev, A.I. Rodin, V.V. Yakunin, A.D. Bogatov, V.S. Bochkin, A.M. Chegodajkin // Magazine of Civil Engineering. - 2018. - P. 219-227.

6. Ланина Т.Д. Комплексная утилизация нефтегазопромышленных отходов для обеспечения экологической безопасности и дополнительного извлечения минерального сырья: автореф. дис. ... д-ра техн. наук. - Ухта, 2009. - 34 с.

7. Foo K.Y., Hameed B.H. Utilization of biodiesel waste as a renewable resource for activated carbon: application to environmental problems // Renewable and Sustainable Energy Reviews. - 2009. V. 13. - Iss. 9. - P. 2495-2504.

8. Bolden J., Taher Abu-Lebdeh, Fini E. Utilization of recycled and waste materials in various construction applications // American Journal of Environmental Science. - 2013. - V. 9. - P. 14-24.

9. Plastic recycling in additive manufacturing: a systematic literature review and opportunities for the circular economy / F.A. Cruz Sanchez, H. Boudaoud, M. Camargo, J.M. Pearce // Journal of Cleaner Production. - 2020. - V. 264. - 121602.

10. Assessing the impact of industrial waste on environment and mitigation strategies: a comprehensive review / Vivek Kumar Gaur, Poonam Sharma, Ranjna Sirohi, Mukesh Kumar Awasthi, ClaudeGilles Dussap, Ashok Pandey // Journal of Hazardous Materials. 2020. - V. 398. - 123019.

11. Choudhary J., Kumar B., Gupta A. Utilization of solid waste materials as alternative fillers in asphalt mixes: a review // Construction and Building Materials. - 2020. - V. 234. - 117271.

12. Abdulgazi Gedik. A review on the evaluation of the potential utilization of construction and demolition waste in hot mix asphalt pavements // Resources, Conservation and Recycling. - 2020. V. 161. - 104956

13. White G. State of the art: asphalt for airport pavement surfacing // International Journal of Pavement Research and Technology. 2018. - V. 11. - P. 77-98

14. Gagnon J. Runway grooving - the past, present and future // 8th International Conference on Maintenance and Rehabilitation of Pavements. - Singapore, 2016. - P. 27-29.

15. Zelelew H.M., Papagiannakis A.T. Interpreting asphalt concrete creep behavior through non-Newtonian mastic rheology // Road Materials and Pavement Design. - 2012. - V. 13. - P. 266-278.
16. Pérez-Jiménez F.P., Miró Recasens R., Martínez A. Effect of the nature and filler content on the behavior of the bituminous mastics // Road Materials and Pavement Design. - 2008. - V. 9. - P. 417-431.

17. Influence of filler-bitumen ratio on performance of modified asphalt mortar by additive / Hongsheng Qiu, Ximing Tan, Shu Shi, Heng Zhang // Journal of Modern Transportation. - 2013. - V. 21. P. 40-46.

18. Effect of mineral filler characteristics on asphalt mastic and mixture rutting potential / H. Wang, I.L. Al-Qadi, A.F. Faheem, H.U. Bahia, Shih-Hsien Yang, G.H. Reinke // Transportation Research Record - 2011 - V. 2208 - P. 33-39

19. Liao M.C., Airey G., Chen J.S. Mechanical properties of fillerasphalt mastics // International Journal of Pavement Research and Technology. - 2013. - V. 6. - P. 576-581.

20. Speight J.C. Petroleum asphaltenes. P. 1. Asphaltenes, resins and the structure of petroleum // Oil \& Gas Science and Technology. 2004. - V. 59. - P. 467-477.

21. Holýa M., Remisova E. Analysis of influence of bitumen composition on the properties represented by empirical and viscosity test // Transportation Research Procedia. - 2019. - V. 40. - P. 34-41.

22. Investigation of sterol chemistry to retard the aging of asphalt binders / G. Reinke, G. Baumgardner, A. Hanz, S. King // Transportation Research Record. - 2017. - V. 2633. - Iss. 1. - P. 127 135

23. Alternative approach toward the aging of asphalt binder / B. Hofko, F. Handle, L. Eberhardsteiner, M. Hospodka, R. Blab, J. Fussl, H. Grothe // Transportation Research Record. - 2015. - V. 2505. P. 24-31.

24. Bitumen ageing - impact of reactive oxygen species / B. Hofko, D. Maschauer, D. Steiner, J. Mirwald, H. Grothe // Case Studies in Construction Materials. - 2020. - V. 13. - e00390.

25. Соломенцев А.Б. Классификация и номенклатура модифицирующих добавок для битумов // Наука и техника в дорожной отрасли. - 2008. - № 1. - С. 14-16.

26. Галдина В.Д. Модифицированные битумы. - Омск: СибАДИ, 2009. $-228 \mathrm{c}$

27. Галдина В.Д. Влияние полимерных добавок на свойства битума и асфальтобетона // Вестник СибАДИ. - 2009. - № 12. C. $32-36$.

Поступила 10.03.2021 2.

\section{Информация об авторах}

Дюрягина А.Н., кандидат химических наук, ассоциированный профессор, заведующая кафедрой химии и химических технологий НАО Северо-Казахстанский университет им. М. Козыбаева.

Бызова Ю.С., докторант кафедры химии и химических технологий НАО Северо-Казахстанский университет им. М. Козыбаева.

Островной К.A., магистр, старший преподаватель кафедры химии и химических технологий НАО СевероКазахстанский университет им. М. Козыбаева.

Тюканько В.Ю., кандидат технических наук, $\mathrm{PhD}$, главный технолог TOO «AVAGRO». 
UDC 628.477 .6

\title{
UTILIZATION OF THE WASTE SEALING LIQUID COMPONENT IN ASPHALT CONCRETE PAVEMENTS
}

\author{
Antonina N. Dyuryagina ${ }^{1}$, \\ adyuryagina@inbox.ru \\ Yulia S. Byzova ${ }^{1}$, \\ yuliyabyzovva@gmail.com
}

Kirill A. Ostrovnoy',
kostrovnoy@mail.ru

Vitaliy Y. Tyukanko², vetal3333@mail.ru

${ }^{1}$ NC JSC North Kazakhstan University named after M. Kozybaev, 86, Pushkin street, Petropavlovsk, 150000, Kazakhstan.

2 JSC «AVAGRO»,

122, Novaya street, Petropavlovsk, 150000, Kazakhstan.

The relevance of this research is dictated by the need for solutions in the field of recycling various industrial wastes in order to use them as part of a new product. The object of research is the spent sealant AG-4I, which is used in large volumes at energy facilities, oil and gas production enterprises, machine-building plants, municipal utilities and other industries in heating systems. Recycling saves natural resources, reduces solid waste, air and water pollution, and harmful emissions to the atmosphere. The road construction industry can use recycled materials as part of asphalt pavements. Modification of asphalt concrete mixes with industrial waste is a priority in order to reduce the huge amount of waste generated from various sources.

The aim of this work was to study the possibility of recycling the spent sealing liquid AG-4I as part of asphalt concrete compositions for modification.

Methods: rheological methods, structural and mechanical methods for evaluating the quality of films and coatings.

Results. The authors have determined the physicochemical and rheological characteristics of the spent sealing liquid in the bitumen compositions. As a result of the research, it was found that the preservation of the test sealing liquid in the preferred solid form is impossible, since attempts to polymerize polyisobutylene in the composition of the sealing liquid waste did not lead to changes in the composition. It is established that the test sealing liquid introduction into asphalt concrete compositions allows it to be used as a stabilizing and hydrophobizing additive. As a result, the adhesion to mineral fillers and the strength of coatings are improved.

\section{Key words:}

Waste utilization, sealing liquid, recycling, asphalt concrete mix, bitumen.

\section{REFERENCES}

1. Sagdeeva G.S., Patrakova G.R. Recycling production and consumption waste using their resource potential. Kazan Technological University Bulletin, 2014, vol. 1, pp. 194-198. In Rus.

2. Pinaev V.E. Sostoyanie i problemy ispolzovaniya promyshlennykh tverdykh otkhodov v Rossii [State and problems of using industrial solid waste in Russia]. Issledovano v Rossii, 2004, vol. 7, pp. 1397-1433.

3. Mazurkin P.M., Soldatova V.A. Modern problems of joint processing of solid household and industrial waste. Modern problems of science and education, 2008, vol. 6, pp. 59-68. In Rus.

4. Kosulina T.P., Kononenko E.A., Tsokur O.S. Utilization of oil sludge by the reagent method and the use of utilization products as secondary material resources. Alternative energy and ecology, 2012, vol. 2, pp. 187-192. In Rus.

5. Erofeev V.T., Rodin A.I., Yakunin V.V., Bogatov A.D., Bochkin V.S. Chegodajkin A.M. Alkali-activated slag binders from rock-wool production wastes. Magazine of Civil Engineering, 2018, pp. 219-227.

6. Lanina T.D. Kompleksnaya utilizatsiya neftegazopromyshlennykh otkhodov dlya obespecheniya ekologicheskoy bezopasnosti dopolnitelnogo izvlecheniya mineralnogo syrya. Avtoreferat Dis. Doct. nauk [Comprehensive utilization of oil and gas industrial waste to ensure environmental safety and additional extraction of mineral raw materials. Dr. Diss. Abstract]. Ukhta, 2009. 34 p.

7. Foo K.Y., Hameed B.H. Utilization of biodiesel waste as a renewable resource for activated carbon: application to environmental problems. Renewable and Sustainable Energy Reviews, 2009, vol. 13, Iss. 9, pp. 2495-2504.
8. Bolden J., Abu-Lebdeh T., Fini E. Utilization of recycled and waste materials in various construction applications. American Journal of Environmental Science, 2013, vol. 9, pp. 14-24.

9. Cruz Sanchez F.A., Boudaoud H., Camargo M., Pearce J.M. Plastic recycling in additive manufacturing: A systematic literature review and opportunities for the circular economy. Journal of Cleaner Production, 2020, vol. 264, art. 121602.

10. Vivek Kumar Gaur, Poonam Sharma, Ranjna Sirohi, Mukesh Kumar Awasthi, Claude-Gilles Dussap, Ashok Pandey. Assessing the impact of industrial waste on environment and mitigation strategies: a comprehensive review. Journal of Hazardous Materials, 2020, vol. 398, art. 123019

11. Choudhary J., Kumar B., Gupta A. Utilization of solid waste materials as alternative fillers in asphalt mixes: a review. Construction and Building Materials, 2020, vol. 234, art.117271.

12. Abdulgazi Gedik. A review on the evaluation of the potential utilization of construction and demolition waste in hot mix asphalt pavements. Resources, Conservation and Recycling, 2020, vol. 161, art. 104956

13. White G. State of the art: asphalt for airport pavement surfacing. International Journal of Pavement Research and Technology, 2018, vol. 11, pp. 77-98.

14. Gagnon J. Runway grooving - the past, present and future. 8th $I n$ ternational Conference on Maintenance and Rehabilitation of Pavements. Singapore, 2016. pp. 27-29.

15. Zelelew H.M., Papagiannakis A.T. Interpreting asphalt concrete creep behavior through non-Newtonian mastic rheology. Road Materials and Pavement Design, 2012, vol. 13, pp. 266-278. 
16. Pérez-Jiménez F.P., Miró Recasens R., Martínez A. Effect of the nature and filler content on the behavior of the bituminous mastics. Road Materials and Pavement Design, 2008, vol. 9, pp. 417-431.

17. Hongsheng Qiu, Ximing Tan, Shu Shi, Heng Zhang. Influence of filler-bitumen ratio on performance of modified asphalt mortar by additive. Journal of Modern Transportation, 2013, vol. 21, pp. 40-46.

18. Wang H., Al-Qadi I.L., Faheem A.F., Bahia H.U., Shih-Hsien Yang, Reinke G.H. Effect of mineral filler characteristics on asphalt mastic and mixture rutting potential. Transportation Research Record, 2011, vol. 2208, pp. 33-39.

19. Liao M.-C., Airey G., Chen J.-S. Mechanical properties of fillerasphalt mastics. International Journal of Pavement Research and Technology, 2013, vol. 6, pp. 576-581.

20. Speight J.C. Petroleum asphaltenes. P. 1. Asphaltenes, resins and the structure of petroleum. Oil \& Gas Science and Technology, 2004, vol. 59, pp. 467-477.

21. Holýa M., Remisova E. Analysis of influence of bitumen composition on the properties represented by empirical and viscosity test. Transportation Research Procedia, 2019, vol. 40, pp. 34-41.
22. Reinke G., Baumgardner G., Hanz A., King S. Investigation of sterol chemistry to retard the aging of asphalt binders. Transportation Research Record, 2017, vol. 2633, Iss. 1, pp. 127-135.

23. Hofko B., Handle F., Eberhardsteiner L., Hospodka M., Blab R., Fussl J., Grothe H. Alternative approach toward the aging of asphalt binder. Transportation Research Record, 2015, vol. 2505, pp. 24-31.

24. Hofko B., Maschauer D., Steiner D., Mirwald J., Grothe H. Bitumen ageing - impact of reactive oxygen species. Case Studies in Construction Materials, 2020, vol. 13, e00390.

25. Solomentsev A.B. Classification and nomenclature of modifying additives for bitumen. Science and technology in the road industry, 2008, vol. 1, pp. 14-16. In Rus.

26. Galdina V.D. Modifitsirovannye bitumy [Modified bitumen]. Omsk, SibADI, 2009. 228 p.

27. Galdina V.D. Influence of polymer additives on the properties of bitumen and asphalt concrete. SibADI Bulletin, 2009, vol. 12, pp. 32-36. In Rus.

Received: 10 March 2021.

\section{Information about the authors}

Antonina N. Dyuryagina, Cand. Sc., associate professor, head of the Department of Chemistry and chemical technologie, NC JSC North Kazakhstan University named after M. Kozybaev.

Yulia S. Byzova, doctoral student, NC JSC North Kazakhstan University named after M. Kozybayev.

Kirill A. Ostrovnoy, master, senior lecturer, NC JSC North Kazakhstan University named after M. Kozybayev.

Vitaliy Y. Tyukanko, Cand. Sc., PhD, chief technologist, JSC «AVAGRO». 